\title{
Phonon and plasmon excitation in inelastic electron tunneling spectroscopy of graphite
}

\author{
L. Vitali, M. A. Schneider, and K. Kern \\ Max-Planck-Institut für Festkörperforschung, Heisenbergstrasse 1, D-70569 Stuttgart, Germany \\ L. Wirtz and A. Rubio \\ Department of Material Physics, University of the Basque Country, Centro Mixto CSIC-UPV, and Donostia International Physics Center, \\ Paseo Manuel de Lardizabal 4, E-20018 San Sebastián, Spain
}

(Received 20 October 2003; revised manuscript received 19 December 2003; published 30 March 2004)

\begin{abstract}
The inelastic electron tunneling spectrum (IETS) of highly oriented pyrolitic graphite has been measured with scanning tunneling spectroscopy (STS) at $6 \mathrm{~K}$. The observed spectral features are in very good agreement with the vibrational density of states of graphite calculated from first principles. We discuss the enhancement of certain phonon modes by phonon-assisted tunneling in STS based on the restrictions imposed by the electronic structure of graphite. We also demonstrate the local excitation of surface plasmons in IETS, which are detected at an energy of $40 \mathrm{meV}$.
\end{abstract}

DOI: 10.1103/PhysRevB.69.121414

PACS number(s): 68.35.Ja, 63.20.-e, 68.37.Ef

Initially applied to measure inelastic tunneling currents across metal-insulator-adsorbate-metal junctions, ${ }^{1}$ inelastic electron tunneling spectroscopy (IETS) has in the recent past been revived and its concept extended to scanning tunneling microscopy (STM-IETS). ${ }^{2}$ Superimposed on the elastic tunneling current, the inelastic tunneling process opens up alternative tunneling channels increasing the total conductance at the onset of its excitation. Very recently, elegant experiments (e.g., Refs. 2-5) have been performed in detecting local inelastic electron energy-loss spectra of individual molecule adsorbed on metallic substrates. The observed features in the tunneling spectra are found to correspond to the vibrational modes of the adsorbed species. Vibrations of single molecules as large as $\mathrm{C}_{60}$ have have been detected. ${ }^{6}$ Despite the increasing number of publications of STM-IETS experiments on single molecules adsorbed on metals, to our knowledge, only two studies were reported on collective vibrational excitations of surfaces. ${ }^{7,8}$ In both these studies graphite was used as a substrate and several spectral features remained unexplained or were attributed to vibrational excitation in the tungsten tip. However, these tip effects have not been detected in many other STM-IETS experiments. In order to resolve the pure inelastic response of a graphite surface, we have performed STM-IETS experiments on a highly oriented pyrolitic graphite crystal (HOPG) at cryogenic temperature. In our experiments the whole vibrational spectrum of HOPG from the rigid layer shear mode at $\approx 5 \mathrm{meV}$ to the optical modes at $\approx 200 \mathrm{meV}$ is clearly observed without being perturbed by inelastic effects from the tip. The phonon modes of graphite can be clearly identified by the direct comparison of experimental data with first-principles density-function theory (DFT) calculations. We report also the observation of surface-plasmon losses with STM-IETS.

Freshly cleaved HOPG has been introduced into the vacuum system and shortly annealed at $500 \mathrm{~K}$. The experiments were performed at $6 \mathrm{~K}$ with a home built UHV-STM using a tungsten tip. The inelastic tunneling spectrum of HOPG was measured over a topographically clean and flat terrace with a lock-in technique. In this technique a sinusoidal signal with amplitude $\Delta V$ is superimposed to the ap- plied sample bias. In IETS the second-harmonic component of the modulated signal, which is proportional to the second derivative versus bias of the tunneling current, $d^{2} I / d V^{2}$, is recorded. In this way the inelastic loss features in the conductance are transformed into peaks at the onset of the loss modes for positive sample bias and into minima for negative sample bias, respectively. A typical example for HOPG is shown in Fig. 1.

Characteristic fingerprint of an inelastic tunneling process is the symmetry with respect to electron and hole tunneling. This is indeed shown in Fig. 1, where features are symmetrically located around the Fermi level (zero bias). However, the amplitude of the signal appears to be bias dependent. Due to this reason, the negative-bias side was chosen for a highresolution study of the $d^{2} I / d V^{2}$ signal shown in Fig. 2(a). The data were obtained with a bias modulation of $\Delta V$ $=10 \mathrm{meV} \mathrm{rms}$ at a frequency of $2.7 \mathrm{kHz}$ and inverted to render the phonon excitations as peaks to facilitate the comparison to theory. Since the electron density of states of HOPG is flat in the energy range close to the Fermi level, the

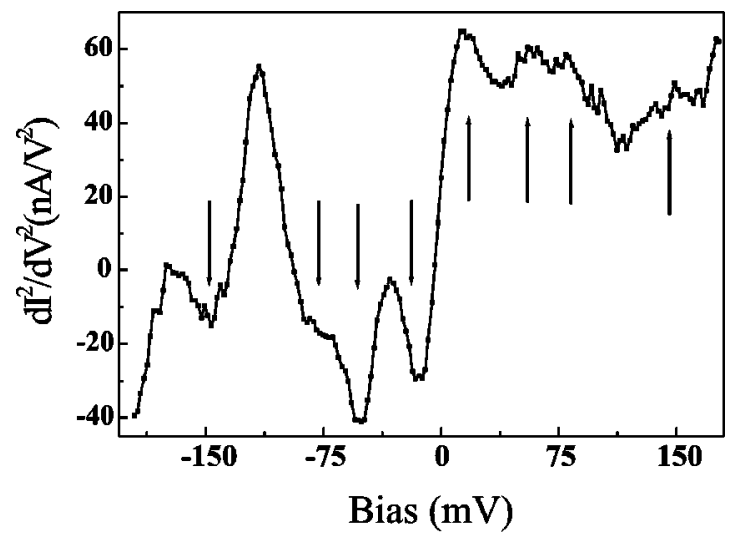

FIG. 1. Inelastic electron tunneling spectrum of HOPG measured by STM spanning a large energy range in both bias polarities. The main features (arrows) in the $d^{2} I / d V^{2}$ are symmetrically located in energy with respect to the zero-bias position. Due to the symmetry they can be assigned to inelastic phonon contributions. 


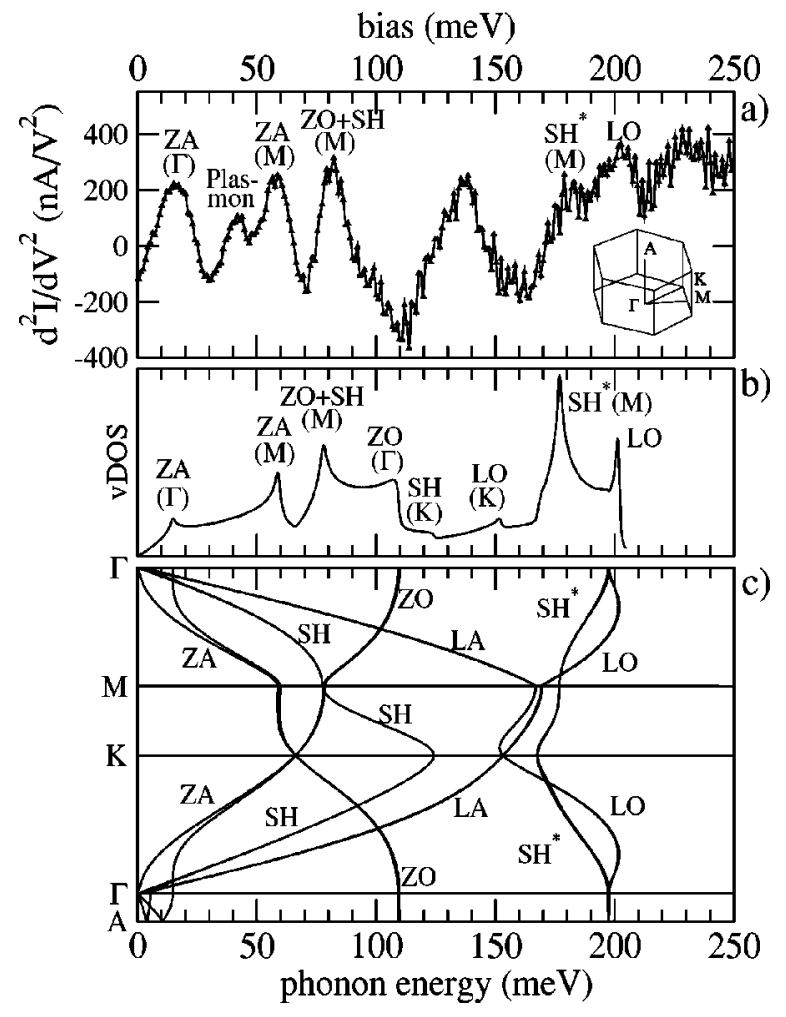

FIG. 2. (a) Inelastic electron tunneling spectrum of HOPG measured by STM. Inelastic excitations show up as peaks in the $d^{2} I / d V^{2}$ signal. (b) Vibrational density of states calculated by DFTLDA. (c) Phonon dispersion relation of graphite. The phonon branches are specified as following: out-of-plane acoustic (ZA), acoustic shear (SH), longitudinal acoustic (LA), out-of-plane optical (ZO), optical shear $\left(\mathrm{SH}^{*}\right)$, and longitudinal optical (LO). The inset shows the first Brillouin zone of graphite with the high symmetry points. A detailed description of the observed phonon modes is given in the text. Furthermore, a plasmon excitation is identified at $40 \mathrm{meV}$.

features in the spectrum in Figs. 1 and 2 are exclusively due to inelastic processes.

Along with the measured inelastic spectrum, we present in Figs. 2(b) and 2(c) the calculated vibrational density of states (vDOS) and the phonon-dispersion relation of graphite along the $A-\Gamma-M-K$ directions. Graphite is a layered structure with strong carbon bonds in the plane and weak van der Waals-like forces between the planes. As a result of this anisotropy, the phonon spectrum of graphite covers a very wide range. We have performed first-principles calculations using DFT in the local-density approximation (LDA). ${ }^{9}$ The aim of this calculation is to resolve some of the deviations in the vDOS calculated by the different recent force-constant parametrizations for graphite ${ }^{12-15}$ and to have a solid foundation for the assignment of the measured peaks. The firstprinciples calculation has been shown ${ }^{16-18}$ to yield very close agreement with phonon-dispersion relations measured by high-resolution electron energy-loss spectroscopy (HREELS) along the $\Gamma-\mathrm{M}$ direction ${ }^{12}$ and along the $\Gamma-\mathrm{K}$ direction. ${ }^{13}$ Only for the acoustic shear mode (SH) it was observed $^{18}$ that at the $M$ point, the HREELS data of Ref. 12 yields an energy which is higher by $25 \mathrm{meV}$ than the $a b$
TABLE I. Observed features in the STM-IET spectrum of HOPG in comparison with vibrational modes observed in experiment [Neutron scattering (Ref. 20), HREELS (Refs. 12,13), and double-resonant Raman scattering (Ref. 19)] and with firstprinciples DFT-LDA calculations.

\begin{tabular}{lcccc}
\hline \hline $\begin{array}{l}\text { Phonon } \\
\text { branch }\end{array}$ & $\begin{array}{c}\text { Symmetry } \\
\text { point }\end{array}$ & $\begin{array}{c}\text { STM-IETS } \\
(\mathrm{meV})\end{array}$ & $\begin{array}{c}\text { Other experiments } \\
(\mathrm{meV})\end{array}$ & $\begin{array}{c}\text { DFT-LDA } \\
(\mathrm{meV})\end{array}$ \\
\hline ZA & $\Gamma$ & 16 & 16 (Ref. 20) & 15 \\
$\mathrm{ZA}$ & $\mathrm{M}$ & 58 & 57 (Ref. 12) & 59 \\
$\mathrm{ZO}$ & $\mathrm{M}$ & 81 & 81 (Ref. 12) & 78 \\
$\mathrm{SH}$ & $\mathrm{M}$ & $100($ Weak) & 100 (Ref. 12) & 77 \\
ZO & $\Gamma$ & $111($ Dip) & 108 (Ref. 13) & 109 \\
LA & $\mathrm{K}$ & 137 & 134 (Ref. 19) & 124 \\
SH* & $\mathrm{M}$ & 180 & 172 (Ref. 12) & 177 \\
LO & $\Gamma$ & 200 & $198-205$ (Ref. 12) & $197-202$ \\
\hline \hline
\end{tabular}

initio value. A similar underestimation of the shear-mode frequency might occur as well at the $K$ point where a mode with energy $134 \mathrm{meV}$ was observed by double-resonance Raman scattering. ${ }^{19}$ Our calculated vDOS is also at variance with the HREELS data at $M$ (Ref. 12) but is in very good agreement with the vDOS measured by neutron scattering on a powdered sample of graphite ${ }^{21}$ where a separate $\mathrm{SH}(M)$ feature was not observed. However, since the SH $K$-point mode contributes only weakly to the calculated DOS, the comparison to the experimental vDOS in Ref. 21 cannot answer the question whether the mode comes out too soft in the calculations.

The observed vibrational spectral features, their comparison with values obtained with other experimental techniques, and their assignment to phonon modes at the various points of the surface Brillouin zone (SBZ) are summarized in Table I. Most features coincide with peaks in the calculated vDOS which arise from the flat dispersion relation around the highsymmetry points. In particular, the higher peaks due to phonon modes around the $M$ point are clearly mapped. The first peak at $16 \mathrm{meV}$ is in good agreement with the calculated energy of the out-of-plane acoustic (ZA) mode at $\Gamma$ where two neighboring planes are oscillating out-of-phase. While in the vDOS and in neutron scattering ${ }^{21}$ this peak is only weak, in the IETS experiment it is strongly pronounced, which can be explained by the direction of oscillation which is orthogonal to the surface plane. The weak shoulder at $6 \mathrm{meV}$ corresponds to the low-frequency horizontal mode at $\Gamma$ where two neighboring planes are oscillating out-of-phase parallel to each other. The prominent feature at $40 \mathrm{meV}$ is not due to a vibrational feature but is assigned to a plasmon excitation (see below). In Ref. 7, a phonon feature at $39 \mathrm{meV}$ was observed, which was associated with a double-phonon excitation in the surface $(16 \mathrm{meV})$ and in the tungsten tip (23 $\mathrm{meV})$. However, in our experiment the tip-phonon peak is clearly not present and all the observed features can therefore be assigned to surface excitation. The vibrational feature observed at $58 \mathrm{meV}$ can be assigned to the $\mathrm{ZA}$ at the $M$ point. The optical out-of-plane (ZO) mode and the shear acoustic mode SH (also called transverse acoustic mode) display a saddle in the dispersion relation around the $M$ point adding up to a very high peak in the vDOS at $78 \mathrm{meV}$. This explains 
the peak at $81 \mathrm{meV}$ in the measurement. However, as noted above, HREELS data ${ }^{12}$ suggest the energy of the SH mode at $M$ to be about $100 \mathrm{meV}$. This gives rise to the alternative explanation that the peak at $81 \mathrm{meV}$ is only due to the $\mathrm{ZO}$ mode while the shoulder at $100 \mathrm{meV}$ may be a signal of the $\mathrm{SH}$ mode at the M-point. The $\mathrm{ZO}$ mode at $\Gamma$ which gives rise to a peak at $108 \mathrm{meV}$ in the theoretical vDOS seems to be missing in the spectrum. Instead, the spectrum reveals a dip $\left(d I^{2} / d V^{2}<0\right)$ at this energy which will be discussed below. At 124 and $150 \mathrm{meV}$ two low-intensity phonon modes, the $\mathrm{SH}$ and the longitudinal acoustic (LA) modes at the $K$ point are predicted, respectively. Experimentally, we have observed a large asymmetric inelastic feature at about $137 \mathrm{meV}$. This disagreement between experiment and theory might point to an underestimation of the SH mode also at the $K$ point. If, as could be the case for the SH mode at $M,{ }^{18}$ the real energy is higher by $10-20 \mathrm{meV}$, the peak at $137 \mathrm{meV}$ can be assigned to the $\mathrm{SH}$ mode at $K$ which corresponds to the above-mentioned observation of a $K$-point phonon at 134 meV (Ref. 19) by double-resonance Raman spectroscopy. In the highest energy range of the spectrum two loss features protrude the background signal at 180 and $200 \mathrm{meV}$, respectively. The first is related to the optical shear mode $\left(\mathrm{SH}^{*}\right.$, also called transverse optical mode) around the $M$ point. The neighboring feature at $200 \mathrm{meV}$ corresponds to the longitudinal optical (LO) branch which displays a strong overbending in the directions $\Gamma-\mathrm{M}$ and $\Gamma-\mathrm{K}$ giving rise to a peak in the vDOS.

In general, the measured IET spectrum follows very closely the vibrational density of states with two notable exceptions which will be discussed in the following. These exceptions are related to the question if all phonons can be excited or if the geometry of the tunneling system leads to a restriction to phonons in a certain region of the Brillouin zone, as it is the case in phonon-assisted electronic tunneling through narrow $p-n$ junctions of semiconductors. In, e.g., $\mathrm{Ge}$, the valence-band maximum is centered at $\Gamma$ while the conduction-band minimum is located at the $L$ point. This allows only vibrational modes at $L$ to be excited by a phonon-assisted tunneling process. ${ }^{22}$ The electronic structure of graphite is also quite unique being a semimetal with a band crossing only at the $K$ point of the SBZ. Therefore the elastic current is carried by electrons that tunnel with a relative large $k_{\|}=K\left(1.7 \AA^{-1}\right)$. In an STM geometry the expansion of the initial state, i.e., the tip wave function in terms of surface plane waves, ${ }^{23}$ leads to a parallel momemtum distribution centered at $k_{\|}=0$. Consequently, the electrons of the elastic current have a low transmission probability. Electrons with any other $k_{\|}$can only tunnel by exchange of finite crystal momentum with a phonon. The phonon-assisted tunneling is relevant to all the dominant features observed besides the $\Gamma$ phonons at 16 and $200 \mathrm{meV}$. An enhanced tunneling contribution should especially occur for electrons with initial parallel momentum $k_{\|}=0$ that tunnel to the Fermi level at the $K$ point by exciting (or absorbing) a $K$-point phonon. This explains why the peak at $137 \mathrm{meV}$ which is probably due to a $K$-point phonon excitation shows up so dominantly in the spectrum even though the vDOS of states only displays weak features for $K$-point phonons.
The second deviation of the measured spectrum from the vDOS of graphite is the dip at $111 \mathrm{meV}$ where the vDOS displays a peak due to the $\mathrm{ZO}$ phonon at $\Gamma$. The theory for inelastic tunneling spectroscopy ${ }^{24,25}$ distinguishes between an inelastic contribution (real phonon emission or absorption) and an elastic contribution (virtual phonon emission with subsequent reabsorption or phonon absorption with subsequent reemission). In some cases, the elastic contribution leads to a decrease of the tunneling conductance $(d I / d V)$ due to backscattering of the electron. ${ }^{24}$ This effect has been measured recently as dips in the $d I^{2} / d V^{2}$ curve of $\mathrm{O}_{2}$ on a silver surface. ${ }^{26}$ We believe that the dip at $111 \mathrm{meV}$ is due to a similar elastic contribution at the onset of vDOS singularity corresponding to the $\mathrm{ZO}(\Gamma)$ phonon branch. In the light of the above discussion of phonon-assisted tunneling on graphite, it is also not surprising that this effect is observed for phonons at $\Gamma$. The only matching pair for a virtual phonon scattering process are electrons initially at $k_{\|}=K$ and phonons at $\Gamma$. All other combinations are forbidden due to the unavailability of intermediate electronic states. Furthermore, the steady reduction of $-d^{2} I / d V^{2}$ from the $\mathrm{ZO}(M)$ peak at $81 \mathrm{meV}$, in contrast to the vDOS, shows that symmetry of the phonon displacement (the ZO branch) should play an important role in the supression of the IET current. ${ }^{27}$ We note that one of the degenerate modes of the $\mathrm{ZO}$ branch at $\Gamma$ is the only $\Gamma$ phonon mode that has $A_{2 u}$ symmetry.

In addition to the predicted phonon modes, the measured spectrum contains an energy-loss feature at $40 \mathrm{meV}$, which cannot be explained by phonon excitations. Other than the previous features, this loss feature is of purely electronic origin and corresponds to an out-of-plane oscillation of the electrons. A tunneling electron traversing a vacuum-metal interface has a significant probability of interacting with collective electron oscillations. The overlapping of the $\pi$ and $\pi^{*}$ band at the $K$ point of the SBZ yields a low density of free carriers (i.e., electrons and holes) near the Fermi level and allows low-energy plasmon excitation. A strong temperature dependence of the excitation energy of this plasmon mode was observed in HREELS. ${ }^{28}$ The excitation energy shifts from $63 \mathrm{meV}$ to $45 \mathrm{meV}$ when going from room temperature to $150 \mathrm{~K}$, respectively. This effect has been attributed to the low density of states at the Fermi level in conjunction with the thermal excitations of carriers. Thermal excitations from the valence into the conduction band can radically alter the electron population at the Fermi level and yields the strong dependence of surface-plasmon energy and linewidth on temperature. The temperature dependence of this excitation energy was also calculated in Ref. 28. Within the random-phase approximation the plasmon energy excitation was predicted to be lowered to $36 \mathrm{meV}$ at $0 \mathrm{~K}$ in rather good agreement with our experimental value. The lowenergy plasmon was also observed in transmission electron energy-loss spectroscopy, which also detected a second plasmon loss, assigned to an excitation polarized parallel to the layer at $128 \mathrm{meV} .{ }^{29}$ The shoulder observed around $125 \mathrm{meV}$ might be assigned to this plasmon excitation.

In conclusion, the IET spectrum of HOPG has been measured by low-temperature scanning tunneling spectroscopy and is compared to an $a b$ initio calculation of the vibrational 
density of states in graphite. The observed vibrational loss features can be identified with peaks in the vDOS which are due to the flat dispersion of the phonon branches around the high-symmetry points of the Brillouin zone. Additionally, a prominent plasmon feature at $40 \mathrm{meV}$ has been identified in the inelastic tunneling current measured with STM. In the past, the local capability of STM-IETS has been proven to be a valuable tool to access the vibrational properties of adsorbates at surfaces. In this work we have shown that
STM-IETS resolves the vibrational and collective electronic excitations of graphite with high accuracy. This is an important step for the application of the method to confined geometries and individual carbon nanostructures.

L.W. and A.R. thank support from the EC research training network COMELCAN (Grant No. HPRN-CT-200000128), Spanish MCyT (Grant No. MAT2001-0946), and UPV/EHU (Grant No. 9/UPV 00206.215-13639/2001). Computer time was provided by CEPBA (Barcelona).
${ }^{1}$ E. L. Wolf, in Tunneling Spectroscopy, edited by P. K. Hansma (Plenum, New York, 1982).

${ }^{2}$ B.C. Stipe, M.A. Rezaei, and W. Ho, Science 280, 1732 (1998).

${ }^{3}$ Y. Kim, T. Komeda, and M. Kawai, Phys. Rev. Lett. 89, 126104 (2002).

${ }^{4}$ H.J. Lee and W. Ho, Science 286, 1719 (1999).

${ }^{5}$ J.I. Pascual et al., Nature (London) 423, 525 (2003).

${ }^{6}$ J.I. Pascual, J. Gomez-Herrero, D. Sanchez-Portal, and H.P. Rust, J. Chem. Phys. 117, 9531 (2002)

${ }^{7}$ D.P.E. Smith, G. Binnig, and C.F. Quate, Appl. Phys. Lett. 49, 1641 (1986).

${ }^{8}$ W. Olejniczak, Z. Klusek, and M. Bieniecki, Appl. Phys. A: Mater. Sci. Process. 66, S191 (1998).

${ }^{9}$ The calculations have been performed with the code ABINIT (Ref. 10). The force constants are calculated within linear-response theory (Ref. 11). The wave functions are expanded in plane waves with an energy cutoff at $80 \mathrm{Ry}$. Core electrons are described by Trouiller-Martins pseudopotentials. The first Brillouin zone is sampled by a $16 \times 16 \times 6$ Monkhorst-Pack grid. The phonon frequencies are converged to within $4 \mathrm{~cm}^{-1}$ with respect to variation of the energy cutoff and variation of $k$-point sampling. The dynamical matrix, which is the Fourier transform of the real-space force constants, is calculated on a $14 \times 14 \times 6$ Monkhorst-Pack grid in the reciprocal space of the phonon-wave vector $q$. From this, the dynamical matrix at any $q$ is obtained by interpolation.

${ }^{10}$ X. Gonze et al., Comput. Mater. Sci. 25, 478 (2002). The ABINIT code is a common project of the Universite Catholique de Louvain, Corning Incorporated and other contributors (URL http:// www.abinit.org).

${ }^{11}$ S. Baroni et al., Rev. Mod. Phys. 73, 515 (2001); X. Gonze, Phys. Rev. B 55, 10337 (1997).
${ }^{12}$ C. Oshima, T. Aizawa, R. Souda, Y. Ishizawa, and Y. Sumiyoshi, Solid State Commun. 65, 1601 (1988); T. Aizawa, R. Souda, S. Otani, Y. Ishizawa, and C. Oshima, Phys. Rev. B 42, 11469 (1990).

${ }^{13}$ S. Siebentritt, R. Pues, K.-H. Rieder, and A.M. Shikin, Phys. Rev. B 55, 7927 (1997).

${ }^{14}$ R. Al-Jishi and G. Dresselhaus, Phys. Rev. B 26, 4514 (1982).

${ }^{15}$ A. Grüneis, R. Saito, T. Kimura, L.G. Cansado, M.A. Pimenta, A. Jorio, A.G. Souta Fiilho, G. Dresselhaus, and M.S. Dresselhaus, Phys. Rev. B 65, 155405 (2002).

${ }^{16}$ G. Kresse, J. Furthmuller, and J. Hafner, Europhys. Lett. 32, 729 (1995).

${ }^{17}$ P. Pavone et al., Physica B 219-220, 439 (1996).

${ }^{18}$ O. Dubay and G. Kresse, Phys. Rev. B 67, 035401 (2003).

${ }^{19}$ Y. Kawashima and G. Katagiri, Phys. Rev. B 52, 10053 (1995).

${ }^{20}$ R. Nicklow, N. Wakabayashi, and H.G. Smith, Phys. Rev. B 5, 4951 (1972).

${ }^{21}$ S. Rols E. Benes, E. Anglaret, J.L. Sauvajol, P. Papenerk, J.E. Fischer, G. Coddens, H. Schober, and A.J. Dianoax, Phys. Rev. Lett. 85, 5222 (2000).

${ }^{22}$ R.T. Payne, Phys. Rev. 139, A570 (1965).

${ }^{23}$ J. Tersoff and D.R. Hamann, Phys. Rev. B 31, 805 (1985).

${ }^{24}$ B.N.J. Persson and A. Baratoff, Phys. Rev. Lett. 59, 339 (1987).

${ }^{25}$ N. Lorente and M. Persson, Phys. Rev. Lett. 85, 2997 (2000).

${ }^{26}$ J.R. Hahn, H.J. Lee, and W. Ho, Phys. Rev. Lett. 85, 1914 (2000).

${ }^{27}$ N. Lorente, M. Persson, L.J. Lauhon, and W. Ho, Phys. Rev. Lett. 86, 2593 (2001).

${ }^{28}$ E.T. Jensen, R.E. Palmer, W. Allison, and J.F. Annett, Phys. Rev. Lett. 66, 492 (1991).

${ }^{29}$ J. Geiger, H. Katterwe, and B. Schröder, Z. Physik 241, 45 (1971). 